\title{
Spitzer + VLTI-GRAVITY Measure the Lens Mass of a Nearby Microlensing Event
}

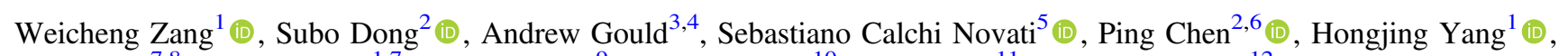

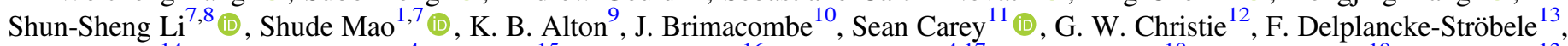
Dax L. Feliz ${ }^{14}$ (D), B. Scott Gaudi ${ }^{4}$, J. Green ${ }^{15}$, Shaoming $\mathrm{Hu}^{16}$, T. Jayasinghe ${ }^{4,17}$ (1), R. A. Koff ${ }^{18}$, A. Kurtenkov ${ }^{19}$, A. Mérand ${ }^{13}$, Milen Minev ${ }^{20}$, Robert Mutel ${ }^{21}$ (i), T. Natusch ${ }^{22}$, Tyler Roth ${ }^{21}$, Yossi Shvartzvald ${ }^{23}$ (ib, Fengwu Sun ${ }^{24}$, T. Vanmunster ${ }^{25}$, and Wei Zhu ${ }^{26}$ (1)

${ }^{1}$ Department of Astronomy and Tsinghua Centre for Astrophysics, Tsinghua University, Beijing 100084, People's Republic of China zangwc17@mails.tsinghua.edu.cn

${ }^{2}$ Kavli Institute for Astronomy and Astrophysics, Peking University, Yi He Yuan Road 5, Hai Dian District, Beijing 100871, People’s Republic of China

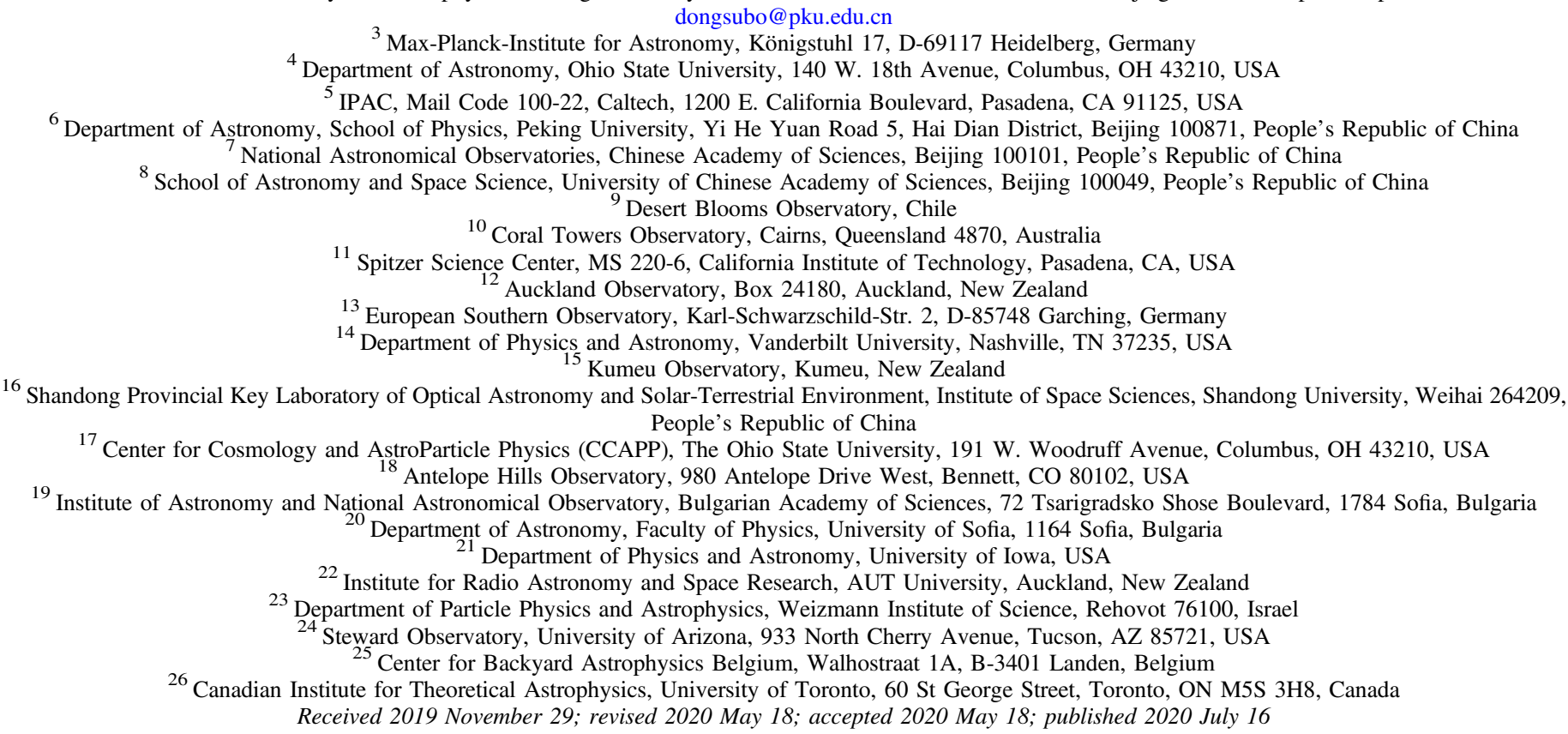
dongsubo@pku.edu.cn

\begin{abstract}
We report the lens mass and distance measurements of the nearby microlensing event TCP J05074264+2447555 (Kojima-1). We measure the microlens parallax vector $\pi_{\mathrm{E}}$ using Spitzer and ground-based light curves with constraints on the direction of lens-source relative proper motion derived from Very Large Telescope Interferometer (VLTI) GRAVITY observations. Combining this $\pi_{\mathrm{E}}$ determination with the angular Einstein radius $\theta_{\mathrm{E}}$ measured by VLTI-GRAVITY observations, we find that the lens is a star with mass $M_{\mathrm{L}}=$ $0.495 \pm 0.063 M_{\odot}$ at a distance $D_{\mathrm{L}}=429 \pm 21 \mathrm{pc}$. We find that the blended light basically all comes from the lens. The lens-source proper motion is $\mu_{\text {rel, hel }}=26.55 \pm 0.36 \mathrm{mas} \mathrm{yr}^{-1}$, so with currently available adaptiveoptics instruments, the lens and source can be resolved in 2021. This is the first microlensing event whose lens mass is unambiguously measured by interferometry + satellite-parallax observations, which opens a new window for mass measurements of isolated objects such as stellar-mass black holes.
\end{abstract}

Unified Astronomy Thesaurus concepts: Gravitational microlensing (672); Satellite microlensing parallax (2148); Stellar masses (1614); Stellar distance (1595)

Supporting material: data behind figure

\section{Introduction}

Gravitational microlensing offers a unique window into probing extrasolar planets beyond the snow line (Mao \& Paczynski 1991; Gould \& Loeb 1992; Gaudi 2012; Mao 2012) and isolated dark objects such as free-floating planets (Sumi et al. 2011; Mróz et al. 2017, 2018, 2019), brown dwarfs (Gould et al. 2009; Shvartzvald et al. 2019), and black holes (Gould 2000a; Bennett et al. 2002; Mao et al. 2002; Wyrzykowski et al. 2016). For a typical microlensing event, the only measured observable that relates to the physical properties of the lens is the Einstein timescale $t_{\mathrm{E}}$. It is a combination of the lens mass $M_{\mathrm{L}}$, the lens-source relative proper motion $\boldsymbol{\mu}_{\text {rel }}$ and parallax $\pi_{\text {rel }}$ by

$$
t_{\mathrm{E}}=\frac{\theta_{\mathrm{E}}}{\mu_{\text {rel }}} ; \quad \theta_{\mathrm{E}}=\sqrt{\kappa M_{\mathrm{L}} \pi_{\text {rel }}} ; \quad \pi_{\text {rel }}=\pi_{\mathrm{L}}-\pi_{\mathrm{S}},
$$

where $\kappa \equiv 4 G /\left(c^{2} \mathrm{AU}\right)=8.144 \mathrm{mas} / M_{\odot}, \theta_{\mathrm{E}}$ is the angular Einstein radius, $\pi_{\mathrm{L}}=\mathrm{AU} / D_{\mathrm{L}}$ and $\pi_{\mathrm{S}}=\mathrm{AU} / D_{\mathrm{S}}$ are the lens and source parallax, respectively, and $D_{\mathrm{L}}$ and $D_{\mathrm{S}}$ are the lens and the source distances, respectively. Therefore, with only $t_{\mathrm{E}}$ 
known, the lens mass and distance cannot be unambiguously determined. By far, the most common way to break this degeneracy is to also measure the angular Einstein radius $\theta_{\mathrm{E}}$ and the microlens parallax $\pi_{\mathrm{E}}$. For a lensing object, its mass is related to these two observables by (Gould 1992, 2000b)

$$
M_{\mathrm{L}}=\frac{\theta_{\mathrm{E}}}{\kappa \pi_{\mathrm{E}}},
$$

and the lens-source relative proper motion and parallax by

$$
\boldsymbol{\mu}_{\mathrm{rel}}=\frac{\theta_{\mathrm{E}}}{t_{\mathrm{E}}} \frac{\boldsymbol{\pi}_{\mathrm{E}}}{\pi_{\mathrm{E}}} ; \quad \pi_{\mathrm{rel}}=\theta_{\mathrm{E}} \pi_{\mathrm{E}}
$$

where the microlens parallax vector $\pi_{\mathrm{E}}$ is defined by

$$
\boldsymbol{\pi}_{\mathrm{E}} \equiv \frac{\pi_{\mathrm{rel}}}{\theta_{\mathrm{E}}} \frac{\boldsymbol{\mu}_{\mathrm{rel}}}{\mu_{\mathrm{rel}}} .
$$

There are three methods to measure the microlens parallax $\pi_{\mathrm{E}}$. The first one is "orbital microlens parallax," which is due to the Earth's orbital acceleration around the Sun, which introduces deviation from rectilinear motion in the lens-source relative motion (Gould 1992). However, this method is generally only feasible for events with long microlensing timescales $t_{\mathrm{E}} \gtrsim$ yr $/ 2 \pi$ (e.g., Udalski et al. 2018) and/or events produced by nearby lenses (e.g., Jung et al. 2018). The second method is "terrestrial microlens parallax" (Hardy \& Walker 1995; Holz \& Wald 1996), which in rare cases can be measured by a combination of simultaneous observations from well-separated ground-based telescopes (e.g., Gould et al. 2009; Yee et al. 2009). The most efficient and robust way to measure $\pi_{\mathrm{E}}$ is via "satellite microlens parallax," which is done by observing the same microlensing event from Earth and one or more wellseparated ( AU) satellite (Refsdal 1966; Gould 1994, 1995). The first observed example applying this method was the event OGLE-2005-SMC-001 (Dong et al. 2007), in which the joint analysis of ground-based observations and Spitzer observations indicates that the lens is probably a halo binary. Microlens parallax measurements from two satellites (Spitzer and the twowheel Kepler K2) have also been achieved to measure the parallax of the event OGLE-2016-BLG-0975 (Zhu et al. 2017b). Since 2014, the Spitzer satellite has observed about 1000 microlensing events toward the Galactic bulge (Gould et al. 2013, 2014, 2015a, 2015b, 2016, 2018), in order to probe planets in substantially different Galactic environments (Calchi Novati et al. 2015a; Zhu et al. 2017a), and they have yielded unambiguous mass measurements for eight planetary systems (Udalski et al. 2015; Street et al. 2016; Shvartzvald et al. 2017; Calchi Novati et al. 2018; Ryu et al. 2018; Jung et al. 2019; Gould et al. 2020; Hirao et al. 2020).

The angular Einstein radius $\theta_{\mathrm{E}}$ is generally measured via finite-source effects (Gould 1994; Nemiroff \& Wickramasinghe 1994; Witt \& Mao 1994) when the source transits a caustic (where the magnification diverges to infinity) or comes close to a cusp. The detection of finite-source effects usually yields the source radius normalized by the Einstein radius, $\rho$, and combined with the source angular radius $\theta_{*}$, which is routinely determined from the intrinsic color and dereddened brightness of the source (Yoo et al. 2004), can lead to the measurement of the angular Einstein radius $\theta_{\mathrm{E}}=\theta_{*} / \rho$. Finite-source effects are frequently detected in binary/planetary events due to their relatively large caustic structures, but they are rarely measured in a single-lens event, since its caustic is a single geometric point.
In addition to the mass measurements from combining the angular Einstein radius $\theta_{\mathrm{E}}$ and the microlens parallax $\pi_{\mathrm{E}}$, an independent mass-distance relationship can be obtained if the flux from the lens system is measured with high angular resolution imaging and compared to stellar models (see, e.g., Bennett et al. 2007; Yee 2015). Accurate lens flux measurement is achieved by resolving the source and lens $\sim 5-20 \mathrm{yr}$ after the microlensing event and thus directly measuring the lens flux (e.g., Alcock et al. 2001; Kozłowski et al. 2007; Batista et al. 2015; Bennett et al. 2015, 2020; Bhattacharya et al. 2018; Vandorou et al. 2019). In addition, resolving the source and the lens can also measure the lens-source relative proper motion $\boldsymbol{\mu}_{\text {rel }}$ by combining this with $t_{\mathrm{E}}$ yields $\theta_{\mathrm{E}}$. The additional mass-distance relationship from the lens flux, combined with the constraints from the angular Einstein radius $\theta_{\mathrm{E}}$ and/or the microlens parallax, can yield the mass and distance of the lens system (e.g., Dong et al. 2009; Beaulieu et al. 2018). However, this method is not feasible for dark lenses such as free-floating planets and black holes.

Here we report the lens-mass measurement of the nearby microlensing event TCP J05074264+2447555 (hereafter referred to as "Kojima-1" for brevity) by a joint analysis of ground-based, Spitzer, and Very Large Telescope Interferometer (VLTI) GRAVITY observations (Gravity Collaboration et al. 2017) of Dong et al. (2019). Nucita et al. (2018) discovered short-lived planetary features due to a super-Earth planet in this event. Fukui et al. (2019) followed it up with Keck AO imaging, and combining it with an upper limit of $\pi_{\mathrm{E}}$ and $\theta_{\mathrm{E}}$ measurements from Dong et al. (2019), they found that the blend was most likely due to the lens star with mass $M_{\mathrm{L}}=0.581 \pm 0.033 M_{\odot}$.

\section{Observations}

Kojima-1, at equatorial coordinates $(\alpha, \delta)_{\mathrm{J} 2000}=(05: 07: 42.72$, $+24: 47: 56.4$, Gaia Collaboration et al. 2018), corresponding to Galactic coordinates $(\ell, b)=(178.76,-9.33)$, was first discovered by the Japanese amateur astronomer Tadashi Kojima (Gunma-ken, Japan) on UT 2017-10-25.688. We use groundbased follow-up data to derive basic single-lens parameters and constrain microlens parallax. To minimize the impact on microlens parallax measurements from possible systematic errors, we choose the ground-based data that were carefully selected and used by Dong et al. (2019), including the data from All-Sky Automatic Survey for Supernovae (ASAS-SN; Shappee et al. 2014), $0.6 \mathrm{~m}$ telescopes at Post Observatory (RP), $0.5 \mathrm{~m}$ Iowa Robotic Telescope (Iowa) at the Winer Observatory (Arizona, USA), $0.4 \mathrm{~m}$ telescope at Auckland Observatory (AO), and the 1.3 m SMARTS telescope (DePoy et al. 2003) at Cerro Tololo Inter-American Observatory (CTIO). We supplement it with CTIO $V$-band data to derive the VHL color-color relation. All the ground-based data were calibrated to standard magnitude systems. For further descriptions of our ground-based data and their availability in digital format, see Appendix A.

For Spitzer, we submitted a Director Discretion Time (DDT) proposal (Dong et al. 2017) to observe the Kojima-1 on 2017 November 7, and it was approved on 2017 November 9. Due to the Sun-angle limit, it did not start taking observations until 2017 December $19\left(\mathrm{HJD}^{\prime}=8107.2, \mathrm{HJD}^{\prime}=\mathrm{HJD}-2,450,000\right)$. The observations ended on 2018 January $23\left(\mathrm{HJD}^{\prime}=8143.7\right)$. In total, 21 data points were taken, all using the $3.6 \mu \mathrm{m}$ channel ( $L$-band) of the IRAC camera. The data were reduced by the method presented by Calchi Novati et al. (2015b). Koshimoto \& 
Bennett (2019) argued that Spitzer microlens parallax measurements by Zhu et al. (2017a) were inconsistent with the Galactic model they adopted and suggested that this inconsistency might be due to systematic errors in Spitzer photometry. Gould et al. (2020) showed that Spitzer systematic effects were at the $\sim 2 \sigma$ level for an event with weak Spitzer signals. Hirao et al. (2020) analyzed an event with stronger Spitzer signals and found marginal systematic effects at less than the $1 \sigma$ level. In Appendix B, we perform similar tests as Gould et al. (2020) and Hirao et al. (2020) to investigate this issue and find that Spitzer systematics is insignificant for our isolated target.

\section{Light-curve Analysis}

Figure 1 shows the Kojima- 1 data together with the best-fit single-lens model. In this section, we analyze the data with a single-lens model. Nucita et al. (2018) reported a short-duration planetary anomaly near the peak of the event, and we discuss the planetary-lens modeling in Appendix $\mathrm{C}$, in order to double check whether the measurements of the microlens parallax $\pi_{\mathrm{E}}$ are affected by the planetary model.

\subsection{Ground-based Data Only}

The single-lens model has three parameters $t_{0}, u_{0}, t_{\mathrm{E}}$ (Paczyński 1986) to calculate the magnification as a function of time $A(t)$ : the time of the maximum magnification $t_{0}$, the impact parameter $u_{0}$ (in units of the angular Einstein radius $\theta_{\mathrm{E}}$ ), and the Einstein radius crossing time $t_{\mathrm{E}}$. For each data set $i$, we introduce two flux parameters $\left(f_{\mathrm{S}, i}, f_{\mathrm{B}, i}\right)$ in order to model the observed flux $f_{i}(t)$ as

$$
f_{i}(t)=f_{\mathrm{S}, i} A(t)+f_{\mathrm{B}, i},
$$

where $f_{\mathrm{S}, i}$ represents the flux of the source star, and $f_{\mathrm{B}, i}$ represents any blended flux that is not lensed in the photometric aperture. In addition, to fit the orbital microlens parallax, we parameterize the microlens parallax effects by $\pi_{\mathrm{E}, \mathrm{N}}$ and $\pi_{\mathrm{E}, \mathrm{E}}$, which are the north and east components of the microlens parallax vector (Gould 2004), respectively. We also fit $u_{0, \oplus}>0$ and $u_{0, \oplus}<0$ solutions to consider the "ecliptic degeneracy" (Jiang et al. 2004; Poindexter et al. 2005). We find $\pi_{\mathrm{E}}=0.13 \pm 0.47$ for the $u_{0, \oplus}>0$ solution and $\pi_{\mathrm{E}}=$ $0.67 \pm 0.52$ for the $u_{0, \oplus}<0$ solution. The likelihood distributions of $\left(\pi_{\mathrm{E}, \mathrm{N}}, \pi_{\mathrm{E}, \mathrm{E}}\right)$ from ground-based data are shown in Figure 2.

\subsection{Satellite Parallax}

For Kojima-1, Spitzer took observations in $1.7<$ $\left(t-t_{0, \oplus}\right) / t_{\mathrm{E}}<3$.1. We can estimate the microlens parallax by

$$
\begin{gathered}
\vec{\pi}_{\mathrm{E}} \sim \frac{\mathrm{au}}{D_{\perp}}(\Delta \tau, \Delta \beta), \quad \Delta \tau \equiv \frac{t_{0, \text { Spitzer }}-t_{0, \oplus}}{t_{\mathrm{E}}}, \\
\Delta \beta \equiv \pm u_{0, \text { Spitzer }}- \pm u_{0, \oplus},
\end{gathered}
$$

where $D_{\perp}$ is the projected separation between the Spitzer satellite $^{27}$ and Earth at the time of the event. Generally, the four possible values of $\Delta \beta$ result in a set of four degenerate solutions (Refsdal 1966; see also Figure 1 from Gould 1994). However, the four solutions merge into two disjoint $\left(u_{0, \oplus}>0\right.$

\footnotetext{
27 We extract the geocentric locations of Spitzer from the JPL Horizons website: http://ssd.jpl.nasa.gov/?horizons.
}

and $\left.u_{0, \oplus}<0\right)$ solutions because the Spitzer data commence

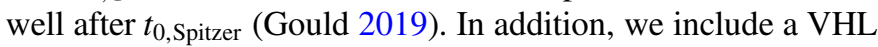
color-color constraint on the Spitzer source flux $f_{s, \text { Spitzer }}$ (e.g., Calchi Novati et al. 2015a), which adds a $\chi_{\text {Color }}^{2}$ into the total $\chi_{\text {total }}^{2}$,

$$
\chi_{\text {Color }}^{2}=\frac{\left[(V-L)_{\mathrm{S}}-(V-L)_{\mathrm{fix}}\right]^{2}}{\sigma_{\mathrm{cc}}^{2}},
$$

where $(V-L)_{\mathrm{S}}$ is the source color from the modeling, $(V-L)_{\text {fix }}$ is the color constraint, and $\sigma_{\mathrm{cc}}$ is the uncertainty of the color constraint. To derive the color constraint, we extract the CTIO $V$ - and $H$ - band and Spitzer $L$-band photometry for stars within $1^{\prime}$ (10 stars in total), and fit for the two parameters $c_{0}$ and $c_{1}$ in the equation

$$
V_{\text {CTIO }}-L_{\text {Spitzer }}=c_{0}+c_{1}\left(V_{\text {CTIO }}-H_{\text {CTIO }}-X_{p}\right) \text {, }
$$

where $X_{p}=3.14$ is a pivot parameter chosen to minimize the covariance between $c_{0}$ and $c_{1}$. We find $c_{0}=2.370 \pm 0.016$, $c_{1}=0.988 \pm 0.043$. We derive the source color by regression of CTIO $V$ versus $H$ flux as the source magnification changes, and find $\left(V_{\text {CTIO }}-H_{\text {CTIO }}\right)_{\mathrm{S}}=2.096 \pm 0.024, \quad(V-L)_{\text {fix }}=$ $1.34 \pm 0.06$. We apply the color constraint to the modeling and find $\pi_{\mathrm{E}}=0.68 \pm 0.23$ for the $u_{0, \oplus}>0$ solution and $\pi_{\mathrm{E}}=1.01 \pm 0.34$ for the $u_{0, \oplus}<0$ solution.

The measured satellite parallax above is a combination of orbital parallax and satellite parallax. To investigate the satellite parallax from the Spitzer data, we fix $\left(t_{0}, u_{0, \oplus}, t_{\mathrm{E}}\right)$ from the best-fit non-parallax model, and fit the parallax only with Spitzer data and the color constraint. We find the resulting "Ground + Spitzer" parallax is an intersection of the satellite parallax, which is nearly a circle, and the orbital parallax, which is nearly a straight line. Gould (2019) shows that the nearly circular shape of the satellite-parallax contours are due to partial overlap of a series of osculating, exactly circular, degeneracies in the $\pi_{\mathrm{E}}$ plane, and similar satellite-parallax shapes have been detected in three Spitzer planetary events (Jung et al. 2019; Gould et al. 2020; Hirao et al. 2020). The best-fit parameters for the $u_{0, \oplus}>0$ and $u_{0, \oplus}<0$ solutions are shown in Tables 1 and 2 , respectively. The likelihood distributions of $\left(\pi_{\mathrm{E}, \mathrm{N}}, \pi_{\mathrm{E}, \mathrm{E}}\right)$ from "Spitzer-only" and "Ground + Spitzer" are shown in Figure 2.

\subsection{VLTI Constraint on the Parallax Direction}

VLTI-GRAVITY provides constraints on the direction of the lens-source relative proper motion and thus (see Equation (4)) the direction of the microlens parallax (see Figure 4 of Dong et al. 2019). For the "no lens light" model, the direction of the microlens parallax (North through East)

$$
\begin{gathered}
\Phi_{\mathrm{VLTI}}=192^{\circ} .9 \pm 0.4 \text { for } u_{0, \oplus}<0, \\
\Phi_{\mathrm{VLTI}}=156^{\circ} .1 \pm 0.4 \text { for } u_{0, \oplus}>0,
\end{gathered}
$$

and for the "luminous lens" model

$$
\begin{aligned}
& \Phi_{\mathrm{VLTI}}=193^{\circ} .5 \pm 0.4 \text { for } u_{0, \oplus}<0, \\
& \Phi_{\mathrm{VLTI}}=156^{\circ} .7 \pm 0.4 \text { for } u_{0, \oplus}>0 .
\end{aligned}
$$



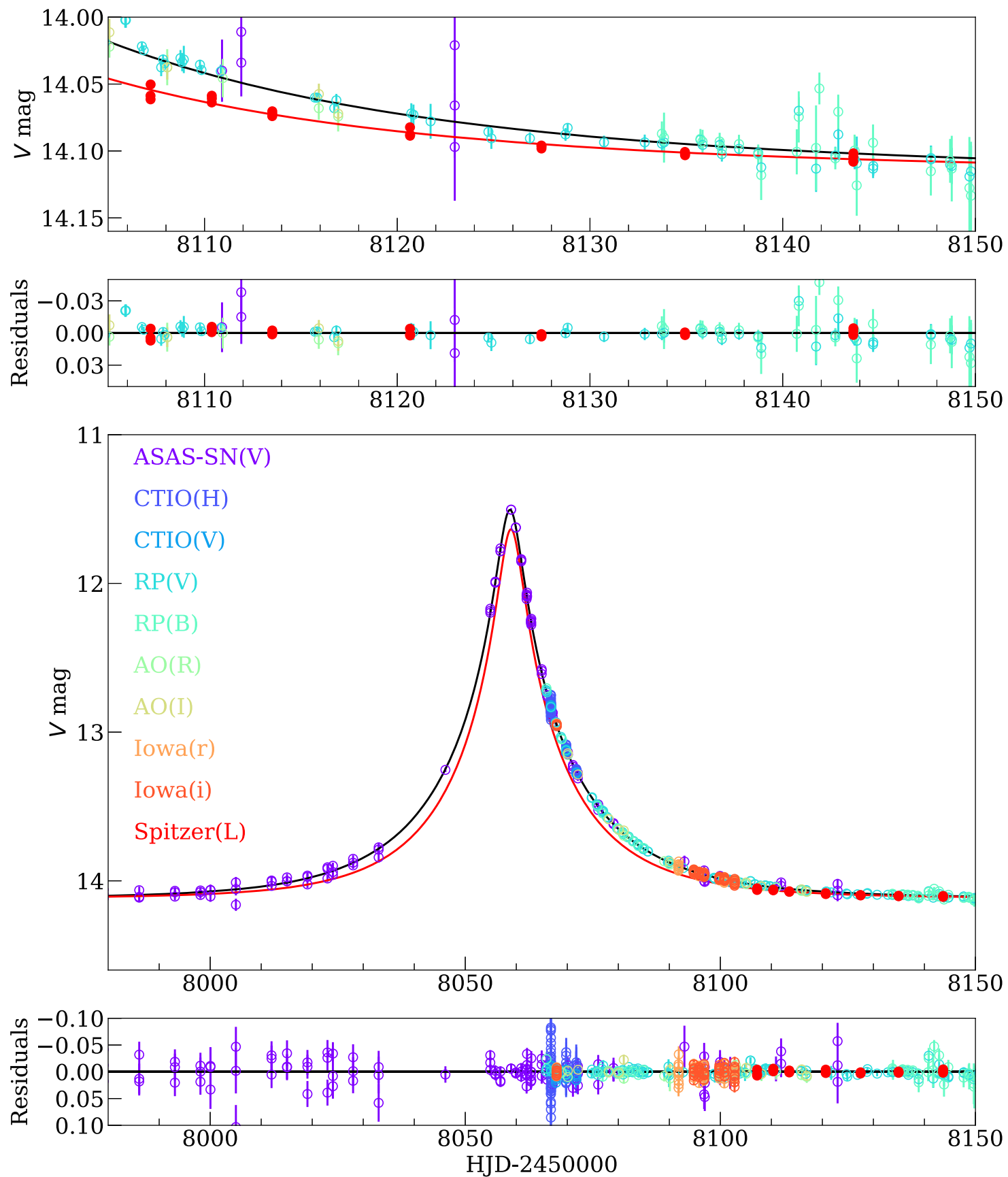

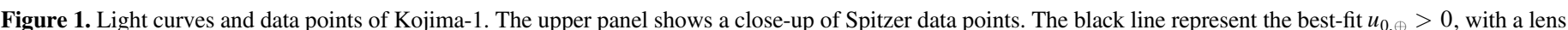

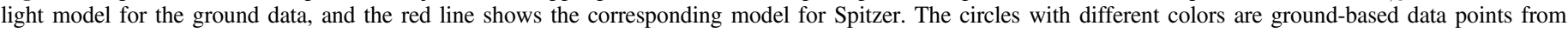
different telescopes or bands. The red dots are Spitzer data points.

(The data used to create this figure are available.)

We include the constraint of the parallax direction by adding a $\chi_{\mathrm{VLTI}}^{2}$ into the total $\chi_{\text {total }}^{2}$,

$$
\chi_{\mathrm{VLTI}}^{2}=\frac{\left(\Phi_{\text {model }}-\Phi_{\mathrm{VLTI}}\right)^{2}}{\sigma_{\mathrm{VLTI}}^{2}},
$$

where $\Phi_{\mathrm{VLTI}}$ is the parallax direction from the modeling, and $\sigma_{\mathrm{VLTI}}=0^{\circ} .4$ is the uncertainty of the VLTI parallax direction. The best-fit parameters are shown in Tables 1 and 2, and its likelihood distributions of $\left(\pi_{\mathrm{E}, \mathrm{N}}, \pi_{\mathrm{E}, \mathrm{E}}\right)$ are shown in Figure 3. For both the "no lens light" model and the "luminous lens" model, the $u_{0, \oplus}<0$ solution is disfavored by $\Delta \chi^{2}>41$. Actually, from the likelihood distributions of $\left(\pi_{\mathrm{E}, \mathrm{N}}, \pi_{\mathrm{E}, \mathrm{E}}\right)$ from "Ground + Spitzer" data shown in Figure 2, we can see that the VLTI parallax direction for the $u_{0, \oplus}<0$ solution is inconsistent with the constraint from the "Ground + Spitzer" data. Thus, we reject the $u_{0, \oplus}<0$ solution. In addition, we 

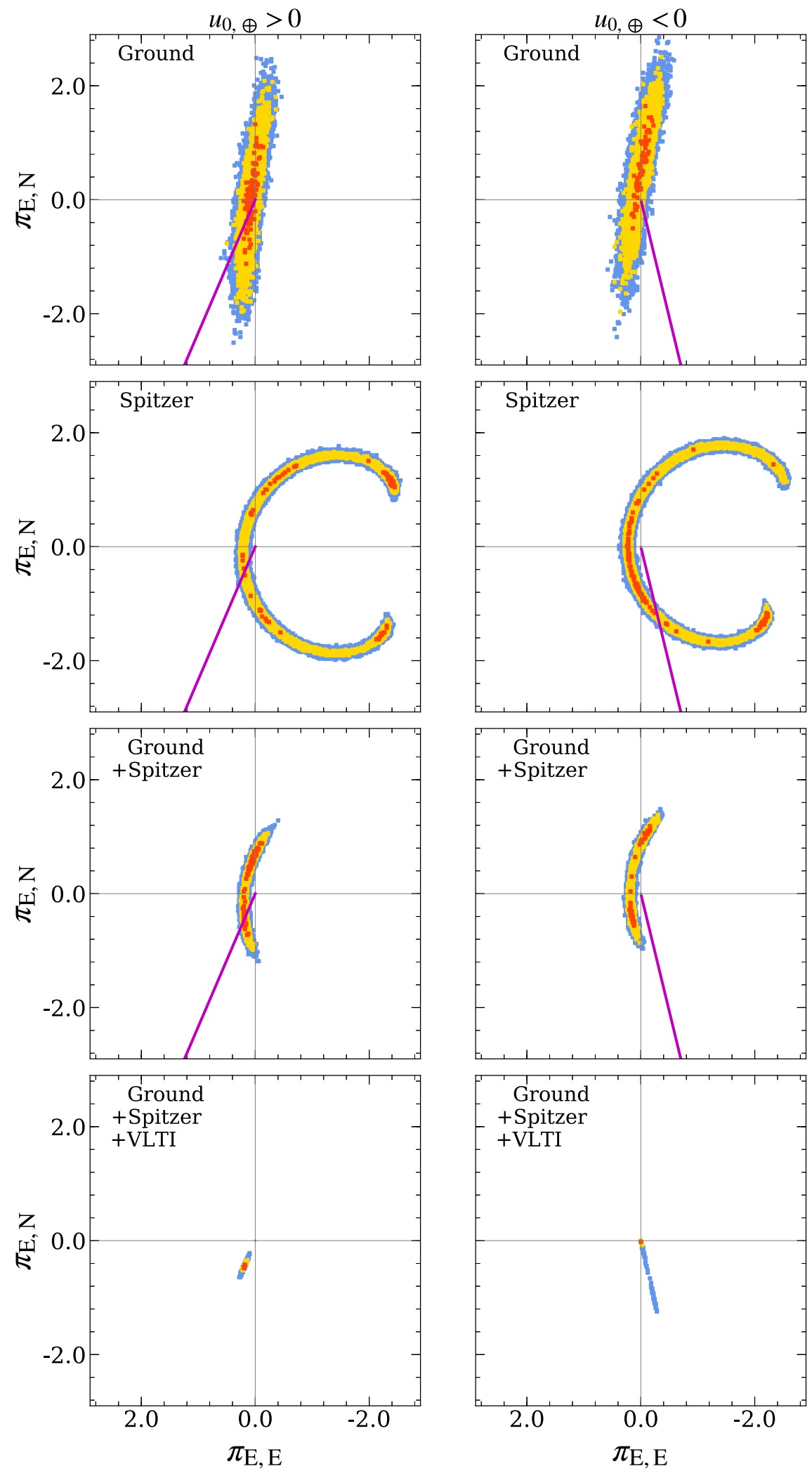

Figure 2. Likelihood distributions for $\pi_{\mathrm{E}}$ derived from MCMC. The left and right panels show the distributions for $u_{0}>0$ and $u_{0}<0$ solutions, respectively. Red, yellow, and blue show likelihood ratios $\left[-2 \Delta \ln \mathcal{L} / \mathcal{L}_{\max }\right]<(1,4, \infty)$, respectively. The magenta lines represent the best value of the VLTI directions.

also check the parallax measurements by including the data in Appendix $\mathrm{A}$ and Fukui et al. (2019). ${ }^{28}$ We find that the

\footnotetext{
${ }^{28} \mathrm{We}$ exclude the data over the planetary signal and only adopt data after $\mathrm{HJD}^{\prime}=8060$.
}

resulting parallax is basically the same with the parallax measured from data used in this paper. Taking the $u_{0, \oplus}>0$ "luminous lens" solution as an example, our result is $\pi_{\mathrm{E}, \mathrm{N}}=-0.430 \pm 0.055, \pi_{\mathrm{E}, \mathrm{E}}=0.186 \pm 0.024$, while the 
Table 1

Best-fit Parameters and Their 68\% Uncertainty Range from MCMC for the $u_{0, \oplus}>0$ Solutions

\begin{tabular}{|c|c|c|c|c|c|}
\hline \multirow[t]{2}{*}{ Parameters } & \multicolumn{5}{|c|}{$u_{0, \oplus}>0$} \\
\hline & Non-parallax & w/o Spitzer & w/ Spitzer & VLTI + Spitzer (w/o Lens Light) & VLTI + Spitzer (w/ Lens Light) \\
\hline$t_{0, \oplus}\left(\mathrm{HJD}^{\prime}\right)$ & $8058.76(1)$ & $8058.76(1)$ & $8058.76(1)$ & $8058.76(1)$ & $8058.76(1)$ \\
\hline$t_{\mathrm{E}}$ & $28.25(15)$ & $27.98(42)$ & $27.89(32)$ & $27.83(15)$ & $27.89(15)$ \\
\hline$\pi_{\mathrm{E}, \mathrm{N}}$ & $\cdots$ & $0.11(83)$ & $0.68(56)$ & $-0.435(56)$ & $-0.430(55)$ \\
\hline$\pi_{\mathrm{E}, \mathrm{E}}$ & $\cdots$ & $0.06(13)$ & $0.01(11)$ & $0.193(24)$ & $0.186(24)$ \\
\hline$V_{\text {СтIO,В }}$ & $17.17(10)$ & $17.38(32)$ & $17.36(27)$ & $17.58(16)$ & $17.55(15)$ \\
\hline$H_{\mathrm{CTIO}, \mathrm{S}}$ & $12.04(1)$ & $12.03(2)$ & $12.03(2)$ & $12.02(1)$ & $12.02(1)$ \\
\hline$H_{\text {СТIO, В }}$ & $13.69(3)$ & $13.74(8)$ & 13.74(7) & $13.79(3)$ & $13.78(3)$ \\
\hline$\chi_{\text {Color }}^{2}$ & $\cdots$ & $\ldots$ & 0.040 & 0.002 & 0.047 \\
\hline$\chi_{\mathrm{VLTI}}^{2}$ & $\cdots$ & $\cdots$ & $\cdots$ & 0.014 & 0.003 \\
\hline
\end{tabular}

resulting parallax including the data in Appendix A and Fukui et al. (2019) is $\pi_{\mathrm{E}, \mathrm{N}}=-0.431 \pm 0.054, \pi_{\mathrm{E}, \mathrm{E}}=0.189 \pm 0.024$.

\section{Physical Parameters}

\subsection{Is the Lens Luminous?}

For the "no lens light" model, VLTI measured the angular Einstein radius $\theta_{\mathrm{E}}=1.850 \pm 0.014$ mas, and the light-curve modeling shows $\pi_{\mathrm{E}}=0.476 \pm 0.061$, so the lens mass $M_{\mathrm{L}}=0.477 \pm 0.061 M_{\odot}$ and the lens-source relative parallax $\pi_{\text {rel }}=\theta_{\mathrm{E}} \pi_{\mathrm{E}}=0.880 \pm 0.113$ mas. The parallax of the "baseline object" has been measured by the Gaia second data release (DR2) (Bailer-Jones et al. 2018; Gaia Collaboration et al. 2018) as

$$
\pi_{\text {base }}=1.480 \pm 0.031 \mathrm{mas}
$$

which is the flux-weighted mean parallax of the source and the blend in Gaia band,

$$
\pi_{\text {base }}=\eta \pi_{\mathrm{B}}+(1-\eta) \pi_{\mathrm{S}}
$$

where $\eta$ is the fraction of total Gaia flux due to the blending, and $\pi_{\mathrm{B}}$ is the parallax of the blending. The Gaia band is qualitatively similar to the $V$ band, and the best-fit solution of the "no lens light" model has $f_{\mathrm{B}, V} / f_{\mathrm{S}, V} \sim 0.04$, so we estimate $\pi_{\mathrm{S}} \sim \pi_{\text {base }}$, and $D_{\mathrm{L}}=\mathrm{AU} /\left(\pi_{\text {rel }}+\pi_{\mathrm{S}}\right)=424 \pm 14$ pc. Using the MIST isochrones ${ }^{29}$ with age $\geqslant 2 \mathrm{Gyr}$, the lens has an absolute $K$-band magnitude of $K_{\mathrm{L}, 0}=5.7 \pm 0.3$. Dong et al. (2019) has found that the source suffers from an extinction $A_{K, \mathrm{~S}}=0.155$, and Fukui et al. (2019) has found the extinction function along the line of sight using Gaia data. We thus estimate the extinction of the lens $A_{K, \mathrm{~L}} \sim 0.136$. As a result, the apparent $K$-band magnitude of the lens is

$$
K_{\mathrm{L}}=K_{\mathrm{L}, 0}+5 \log \frac{D_{\mathrm{L}}}{10 \mathrm{pc}}+A_{K, \mathrm{~L}}=14.0 \pm 0.3,
$$

which is $\sim 12 \%$ of the 2 MASS baseline $K=11.680 \pm 0.018$. Thus, the lens is luminous, and we reject the "no lens light" model.

\footnotetext{
${ }^{29}$ http://waps.cfa.harvard.edu/MIST/interpisos.html
}

\subsection{Blend $=$ Lens?}

Table 3 shows the blend in $H-, I-, R-, V$-bands from the bestfit "luminous lens" model, which shows that the blend is detected in all bands. To estimate the lens apparent brightness, we use the angular Einstein radius $\theta_{\mathrm{E}}=1.891 \pm 0.014$ mas and $\pi_{\mathrm{E}}=0.469 \pm 0.060$ of the "luminous lens" model, and follow the procedure in Section 4.1. For extinction, we adopt the extinction law of Cardelli et al. (1989) and estimate the extinction of the lens using the function of Fukui et al. (2019). The predicted lens apparent magnitude is shown in Table 3. We find that the predicted lens apparent magnitude is consistent with the blend within $1 \sigma$ in all bands. Thus, the blended light basically all comes from the lens.

Fukui et al. (2019) detected no contamination at the position of the microlens on their Keck AO images, and using priors of an upper limit of $\pi_{\mathrm{E}}$ and $\theta_{\mathrm{E}}$ measured by Dong et al. (2019), they found that the majority of the blend is most likely from the lens star rather than an ambient star or a companion to the source or the lens star. Our analysis directly measures the lens mass and distance using measured $\pi_{\mathrm{E}}$ and $\theta_{\mathrm{E}}$, thus it is a direct demonstration that the blend mostly comes from the lens. Note that the VLTI analysis did not consider the possibilities of a companion to the source or the lens, raising a possible concern on whether such scenarios could compromise our argument. From the VLTI analysis, the "no lens light" and the "luminous lens" models result in almost identical $\theta_{\mathrm{E}}(2 \%$ difference) and $\Phi_{\text {VLTI }}(0.6 \mathrm{deg}$ difference $)$, suggesting that they are robustly measured by VLTI regardless of the assumption on the blend. Because these two parameters are the only pieces of information we have used from the VLTI analysis, our measurements of the lens mass and distance are robust. At the $1 \sigma$ level, there can be up to $\sim 33 \%$ of the blend due to a possible companion to the lens or the source, so this represents up to $\sim 2 \%$ of the contribution to the total light during the VLTI observation. We perform simulations to mimic a source/lens companion by randomly placing $2 \%$ of the light within VLTI FOV, and the resulting effects are introducing rms scatters in $\theta_{\mathrm{E}}$ of 0.015 mas and in $\Phi_{\mathrm{VLTI}}$ of $0.36 \mathrm{deg}$, which are slightly larger than their statistical errors $(0.014$ mas and $0.3 \mathrm{deg}$, respectively). Therefore, the possibilities of a source/lens companion have negligible effects in our analysis. 
Table 2

Best-fit Parameters Their 68\% Uncertainty Range from MCMC for the $u_{0, \oplus}<0$ Solutions

\begin{tabular}{|c|c|c|c|c|c|}
\hline \multirow{2}{*}{ Parameters } & \multicolumn{5}{|c|}{$u_{0, \oplus}<0$} \\
\hline & Non-parallax & w/o Spitzer & w/ Spitzer & VLTI + Spitzer (w/o Lens Light) & VLTI + Spitzer (w/ Lens Light) \\
\hline$\overline{t_{0, \oplus}\left(\mathrm{HJD}^{\prime}\right)}$ & $8058.76(1)$ & $8058.76(1)$ & $8058.76(1)$ & $8058.76(1)$ & $8058.76(1)$ \\
\hline$u_{0, \oplus}$ & -0.083 & $-0.084(1)$ & $-0.084(1)$ & $-0.084(1)$ & $-0.082(1)$ \\
\hline$t_{\mathrm{E}}$ & $28.26(16)$ & $28.11(45)$ & $28.24(38)$ & $28.06(35)$ & $28.15(29)$ \\
\hline$\pi_{\mathrm{E}, \mathrm{N}}$ & $\cdots$ & $0.67(81)$ & $1.01(63)$ & $-0.003(234)$ & $-0.003(223)$ \\
\hline$\pi_{\mathrm{E}, \mathrm{E}}$ & $\cdots$ & $-0.01(16)$ & $-0.07(13)$ & $-0.001(50)$ & $-0.001(51)$ \\
\hline$\pi_{\mathrm{E}}$ & $\cdots$ & $0.67(52)$ & $1.01(34)$ & $0.003(239)$ & $0.003(229)$ \\
\hline$V_{\mathrm{CTIO}, \mathrm{S}}$ & $14.18(1)$ & $14.16(2)$ & $14.17(2)$ & $14.17(1)$ & $14.17(1)$ \\
\hline$V_{\text {CTIO,B }}$ & $17.17(10)$ & $17.40(34)$ & $17.33(29)$ & $17.27(27)$ & $17.22(23)$ \\
\hline$H_{\mathrm{CTIO}, \mathrm{S}}$ & $12.04(1)$ & $12.02(2)$ & $12.03(2)$ & $12.03(1)$ & $12.04(1)$ \\
\hline$H_{\mathrm{CTIO}, \mathrm{B}}$ & $13.69(3)$ & $13.75(8)$ & $13.73(7)$ & $13.71(6)$ & $13.70(6)$ \\
\hline$\chi_{\text {Color }}^{2}$ & $\cdots$ & $\cdots$ & 0.002 & 39.840 & 40.728 \\
\hline$\chi_{\mathrm{VLTI}}^{2}$ & $\cdots$ & $\cdots$ & $\cdots$ & 0.033 & 0.009 \\
\hline$\chi_{\text {total }}^{2} /$ dof & $557.83 / 558$ & $556.7 / 556$ & $576.2 / 575$ & $618.2 / 575$ & $618.0 / 575$ \\
\hline
\end{tabular}

\subsection{Lens Parameters}

According to the best-fit "luminous lens" model, the lens mass

$$
M=\frac{\theta_{\mathrm{E}}}{\kappa \pi_{\mathrm{E}}}=0.495 \pm 0.063 M_{\odot},
$$

the lens-source relative parallax

$$
\pi_{\text {rel }}=\theta_{\mathrm{E}} \pi_{\mathrm{E}}=0.887 \pm 0.114 \text { mas. }
$$

Combining Equations (1), (14) and (15), we obtain

$$
\begin{aligned}
\pi_{\mathrm{L}} & =\pi_{\text {base }}+(1-\eta) \pi_{\text {rel }}=2.332 \pm 0.114 \text { mas; } \\
D_{\mathrm{L}} & =\frac{\mathrm{AU}}{\pi_{\mathrm{L}}}=429 \pm 21 \mathrm{pc},
\end{aligned}
$$

where we adopt $\eta=f_{\mathrm{B}, V} /\left(f_{\mathrm{S}, V}+f_{\mathrm{B}, V}\right)=0.040 \pm 0.016$ from the best-fit "luminous lens" model as the fraction of total Gaia flux due to the lens. The geocentric and heliocentric relative proper motion are

$$
\begin{aligned}
& \boldsymbol{\mu}_{\text {rel,geo }}(N, E)=\frac{\theta_{\mathrm{E}}}{t_{\mathrm{E}}} \frac{\boldsymbol{\pi}_{\mathrm{E}}}{\pi_{\mathrm{E}}} \\
& \quad=(-22.73,9.83) \pm(0.21,0.19) \mathrm{mas} \mathrm{yr}^{-1} \\
& \boldsymbol{\mu}_{\text {rel,hel }}(N, E)=\boldsymbol{\mu}_{\text {rel,geo }}+\frac{\pi_{\text {rel }}}{\mathrm{AU}} \boldsymbol{v}_{\oplus, \perp} \\
& =(-22.45,14.18) \pm(0.21,0.59) \mathrm{mas} \mathrm{yr}^{-1},
\end{aligned}
$$

where $\boldsymbol{v}_{\oplus, \perp}=(1.47,23.29) \mathrm{km} \mathrm{s}^{-1}$ is Earth's projected velocity on the event at $t_{0}$. The proper motion of the "baseline object" has been measured by Gaia as

$$
\begin{gathered}
\boldsymbol{\mu}_{\text {base }}(N, E)=(-7.330,-0.228) \\
\pm(0.033,0.061) \mathrm{mas} \mathrm{yr}^{-1},
\end{gathered}
$$

which is also the flux-weighted mean proper motion. Thus, the lens and source proper motion are

$$
\begin{aligned}
& \boldsymbol{\mu}_{\mathrm{L}}(N, E)=\boldsymbol{\mu}_{\text {base }}+(1-\eta) \boldsymbol{\mu}_{\text {rel, hel }} \\
& \quad=(-28.89,13.39) \pm(0.41,0.61){\mathrm{mas} \mathrm{yr}^{-1}} \\
& \boldsymbol{\mu}_{\mathrm{S}}(N, E)=\boldsymbol{\mu}_{\text {base }}-\eta \boldsymbol{\mu}_{\text {rel,hel }} \\
& \quad=(-6.43,-0.80) \pm(0.36,0.23){\mathrm{mas} \mathrm{yr}^{-1}}^{-1}
\end{aligned}
$$

We summarize the derived lens parameters in Table 4.

\section{Discussion and Conclusion}

We have reported the analysis of the microlensing event Kojima-1. The combination of the angular Einstein radius $\theta_{\mathrm{E}}$ measured by VLTI-GRAVITY observations, and the direction of microlens parallax $\pi_{\mathrm{E}}$ constrained from VLTI-GRAVITY, Spitzer, and ground-based observations, reveals that the lens is a $M_{\mathrm{L}}=0.495 \pm 0.063 M_{\odot}$ star at $D_{\mathrm{L}}=429 \pm 21 \mathrm{pc}$. We also note that Fukui et al. (2019) reported the mass and distance of Kojima-1L to be $M_{\mathrm{L}}=0.581 \pm 0.033 M_{\odot}$ star at $D_{\mathrm{L}}=$ $505 \pm 47 \mathrm{pc}$, which is a combination of lens flux from a Keck AO image, source distance from spectroscopy, and $\theta_{\mathrm{E}}$ from VLTI observations (see Table 4 and Figure 9 of Fukui et al. 2019). That is, their mass estimate is $1.2 \sigma$ higher than ours. We note that the main discrepancy between Fukui et al. (2019) and our result comes from the adopted source distances. Fukui et al. (2019) used spectroscopy to determine the source distance and found $D_{\mathrm{S}}=800 \pm 130 \mathrm{pc}$, while we adopt Gaia parallax measurement, which yields the source distance as $D_{\mathrm{S}}=$ $660 \pm 15$ pc. If we adopt the Gaia parallax, $\theta_{\mathrm{E}}$ measured by VLTI-GRAVITY observations and the lens flux taken from Keck AO image Fukui et al. (2019), we find $M_{\mathrm{L}}=0.527 \pm 0.032 M_{\odot}$ star at $D_{\mathrm{L}}=434 \pm 12 \mathrm{pc}$, which is in agreement with our measurements within $1 \sigma$. In Table 5, we summarize the mass and distance of the lens from different measurements. Using current $\mathrm{AO}$ instruments, Batista et al. (2015), Bennett et al. (2015), and Bhattacharya et al. (2018) resolved the lens and source for cases that these have approximately equal brightness when they were separated by 34-60 mas. In this case, the lens-source proper motion is $\mu_{\text {rel, hel }}=26.55 \pm 0.36$ mas yr $^{-1}$, and the lens is about $1.8 \mathrm{mag}$ fainter than the source in the $H$-band. We estimate that it will probably require $\sim 80$ mas to resolve the source and lens. Thus, the derived physical parameters of our work can be verified by currently available AO instruments in 2021 and later.

This is the first microlensing event whose lens mass has been unambiguously measured by interferometry (VLTI-GRAVITY) + satellite (Spitzer) parallax observations. Interferometry + satellite parallax is a new method to measure the mass of isolated objects. The detection frequency of finite-source effects in a single event is only $\sim 2 \%$ (Zhu et al. 2016; 


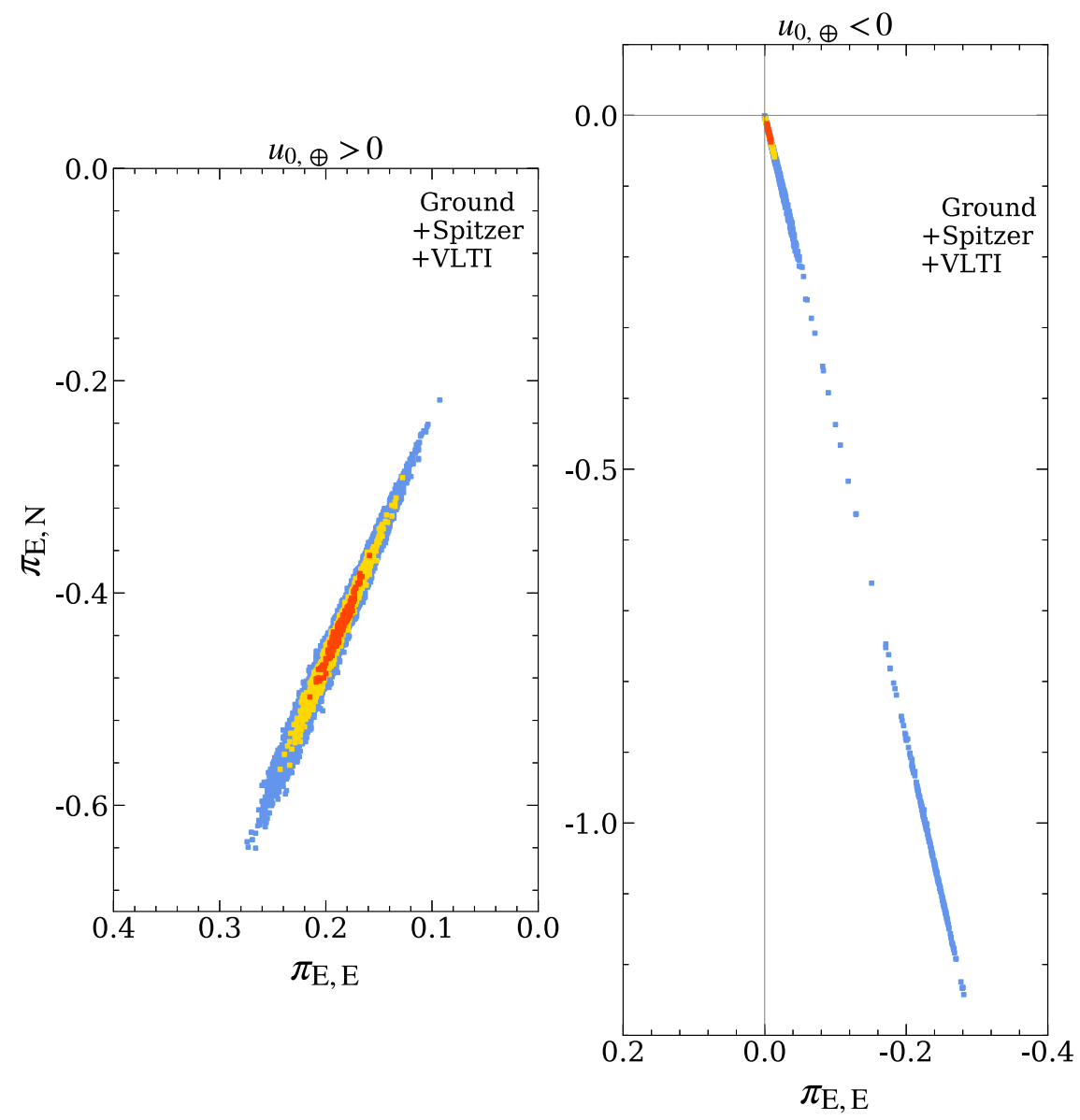

Figure 3. Close-up of $\pi_{\mathrm{E}}$ likelihood distributions with Ground + Spitzer + VLTI constraints. The symbols are similar to those in Figure 2. Note that the best-fit solution shown in the left panel is preferred over that shown in the right panel by $\Delta \chi^{2} \sim 41$.

Table 3

Blend from the Best-fit "Luminous Lens" Model versus the Predicted Apparent Magnitude of the Lens

\begin{tabular}{lcccc}
\hline \hline Band & $H$ & $I$ & $R$ \\
\hline Extinction $A_{\lambda}$ & 0.15 & 0.58 & 0.92 & 1.21 \\
Blending & $13.78 \pm 0.03$ & $16.30 \pm 0.07$ & $16.76 \pm 0.10$ & $17.55 \pm 0.15$ \\
Predicted lens apparent magnitude & $14.0 \pm 0.3$ & $16.4 \pm 0.4$ & $17.0 \pm 0.6$ & $17.9 \pm 0.8$ \\
\hline
\end{tabular}

Table 4

Physical Parameters for Kojima-1L

\begin{tabular}{lr}
\hline \hline$M_{\mathrm{L}}\left(M_{\odot}\right)$ & $0.495 \pm 0.063$ \\
\hline$D_{\mathrm{L}}[\mathrm{pc}]$ & $429 \pm 21$ \\
$D_{\mathrm{S}}[\mathrm{pc}]$ & $692 \pm 15$ \\
$\mu_{\mathrm{L}, \mathrm{hel}, \mathrm{N}}\left[\mathrm{mas} \mathrm{yr}^{-1}\right]$ & $-28.89 \pm 0.41$ \\
$\mu_{\mathrm{L}, \mathrm{hel}, \mathrm{S}}\left[\mathrm{mas} \mathrm{yr}^{-1}\right]$ & $13.39 \pm 0.61$ \\
$\mu_{\mathrm{S}, \mathrm{hel}, \mathrm{N}}\left[\mathrm{mas} \mathrm{yr}^{-1}\right]$ & $-6.42 \pm 0.36$ \\
$\mu_{\mathrm{S}, \mathrm{hel}, \mathrm{S}}\left[\mathrm{mas} \mathrm{yr}^{-1}\right]$ & $-0.80 \pm 0.23$ \\
$\mu_{\text {rel,hel, N }}\left[\mathrm{mas} \mathrm{yr}^{-1}\right]$ & $-22.45 \pm 0.21$ \\
$\mu_{\text {rel,hel, E }}\left[\mathrm{mas} \mathrm{yr}^{-1}\right]$ & $14.18 \pm 0.59$ \\
$\mu_{\text {rel, geo, N }}\left[\mathrm{mas} \mathrm{yr}^{-1}\right]$ & $-22.73 \pm 0.21$ \\
$\mu_{\text {rel,geo, E }}\left[\mathrm{mas} \mathrm{yr}^{-1}\right]$ & $9.83 \pm 0.19$ \\
\hline
\end{tabular}

Zang et al. 2020), so interferometric observation such as VLTIGRAVITY is a complementary approach to measurements of the angular Einstein radius $\theta_{\mathrm{E}}$. In addition, interferometry can
Table 5

Mass and Distance for Kojima-1L Using Different Observables

\begin{tabular}{lccc}
\hline \hline Observables & $\begin{array}{c}\pi_{\mathrm{E}} \text { and } \theta_{\mathrm{E}} \text { and } \\
\text { Gaia Parallax } \\
\text { (This work) }\end{array}$ & $\begin{array}{c}\text { Lens Flux and } \theta_{\mathrm{E}} \\
\text { and Gaia Parallax }\end{array}$ & $\begin{array}{c}\text { Lens Flux and } \theta_{\mathrm{E}} \\
\text { and } D_{\mathrm{S}} \text { from } \\
\text { Spectroscopy } \\
\text { (Fukui et al. 2019) }\end{array}$ \\
\hline$M_{\mathrm{L}}\left[M_{\odot}\right]$ & $0.495 \pm 0.063$ & $0.527 \pm 0.032$ & $0.586 \pm 0.033$ \\
$D_{\mathrm{L}}[\mathrm{pc}]$ & $429 \pm 21$ & $434 \pm 12$ & $505 \pm 47$ \\
\hline
\end{tabular}

provide additional constraints on the microlens parallax direction, which is helpful for breaking degeneracy in the parallax measurements (Refsdal 1966; Gould 1994). Gould (2000a) estimated that $\sim 20 \%$ of Galactic microlensing events are caused by stellar remnants, and specifically that $\sim 1 \%$ are due to stellar-mass black hole lenses. Some black hole candidates (Bennett et al. 2002; Mao et al. 2002; Wyrzykowski et al. 2016) have been reported by microlensing surveys, but none of these candidates had finite-source effects. Therefore, 
interferometry observations such as VLTI-GRAVITY + satellite parallax open up a new window for decisively confirming black hole candidates by measuring their masses.

Nucita et al. (2018) reported a planetary companion in the Kojima-1 lens system. Using the derived lens parameters, we infer the planet mass to be

$$
M_{\text {planet }}=19.0 \pm 3.0 M_{\oplus} .
$$

The projected planet-host separation is

$$
a_{\perp}=s \theta_{\mathrm{E}} D_{\mathrm{L}}=0.78 \pm 0.05 \mathrm{AU},
$$

where $s$ is the planet-host projected separation in units of $\theta_{\mathrm{E}}$. Following the procedure of Han et al. (2019), we estimate the radial-velocity (RV) amplitude $v \sin (i) \sim 2.3 \mathrm{~m} \mathrm{~s}^{-1}$ with a period of $\sim 1.3 \mathrm{yr}$, which may be detectable by high-resolution spectrometers such as VLT/Espresso with 4 VLT telescopes. In addition, the snow-line radius of the lens system is $a_{\mathrm{SL}} \sim 2.7\left(M / M_{\odot}\right)=1.3 \mathrm{AU}$ (Kennedy \& Kenyon 2008). Thus, this is the first Neptune within the snow line detected by microlensing.

We thank Tianshu Wang, Jennifer Yee, Akihiko Fukui, and Naoki Koshimoto for fruitful discussions. We are grateful to Robin Leadbeater and Paolo Berardi for making their spectroscopic observations available to us during the observing campaign. S.D. and P.C. are supported by the National Key R\&D Program of China No. 2019YFA0405100 and Projects 11573003 supported by the National Science Foundation of China (NSFC). W.Z., H.Y., S.-S.L., and S.M. acknowledge support by the National Science Foundation of China (grant No. 11821303 and 11761131004). Work by AG was supported by AST-1516842 and by JPL grant 1500811 . A.G. received support from the European Research Council under the European Unions Seventh Framework Programme (FP 7) ERC grant Agreement No. [321035]. This work is based (in part) on observations made with the Spitzer Space Telescope, which is operated by the Jet Propulsion Laboratory, California Institute of Technology under a contract with NASA. Support for this work was provided by NASA through an award issued by JPL/Caltech. Work by S.M.Hu. was supported by the Natural Science Foundation of Shandong province (No. JQ201702), and the Young Scholars Program of Shandong University (No. 20820162003). W.Z. was supported by the Beatrice and Vincent Tremaine Fellowship at CITA.

\section{Appendix A \\ Ground-based Photometric Observations}

We summarize the ground-based photometric observations collected during our observing campaign in Table A1. The ASCII data will be made available upon publication.
Table A1

Ground-based Photometric Observations

\begin{tabular}{lc}
\hline \hline Site & Filter \\
\hline All-Sky Automatic Survey for Supernovae (ASAS-SN) & $V$ \\
Cerro Tololo Inter-American Observatory (CTIO) & $V, H$ \\
Post Observatory (RP) & $B, V$ \\
Auckland Observatory (AO) & $R, I$ \\
Iowa Robotic Telescope (Iowa) & $r, i$ \\
Desert Bloom Observatory (DBO) & $B, V, I$, Clear \\
Coral Towers Observatory (CTO) & Clear \\
Kumeu Observatory (KO) & Wratten12 \\
Weihai (WH) Observatory of Shandong University & $B, V$ \\
Antelope Hill Observatory (ATO) & $B, V$ \\
Bulgarian National Astronomical Observatory (Rozhen) & $B, V$ \\
Peking University 40 cm Telescope of (PFT) & $\mathrm{UHC}$ \\
Center for Backyard Astronomy Belgium Observatory & $V$, Clear \\
(CBABO) & \\
\hline
\end{tabular}

\section{Appendix B \\ Investigation of the Potential Systematics for Spitzer Photometry}

Koshimoto \& Bennett (2019) argued that the microlens parallax measurements of 50 Spitzer events in Zhu et al. (2017a) were inconsistent with the Galactic model they adopted, and they suggested it was due to systematics in Spitzer photometry. Gould et al. (2020) showed that there were $\sim 2 \sigma$ effects on Spitzer parallax measurements caused by Spitzer photometry systematics for the microlensing event KMT-2018-BLG-0029, which had weak Spitzer signals. The causes of the systematic trends in the Spitzer data are not yet fully understood, and Gould et al. (2020) suggested that these were most likely due to the effect of normal field rotation during the Spitzer observing window. Hirao et al. (2020) analyzed OGLE-2017-BLG-0406, which had relatively strong Spitzer signals, and they found that the Spitzer systematic effects were marginal, at less than the $1 \sigma$ level. Here we investigate the potential systematic effects for Kojima- 1 .

We find that there is a field rotation of about 1 degree during the observations and the target was not observed right at the center of the camera, so the effect of field rotation is a little bit larger than that for the Galactic bulge targets. However, Gould et al. (2020) found that the issues may arise due to poorly determined positions of nearby stars that were many magnitudes brighter than the source. For Kojima-1, the target is well isolated.

In addition, to check the effect of field rotation on Spitzer photometry, we do a similar test to Gould et al. (2020), Hirao et al. (2020). We fit parallax by using the first-half Spitzer data $\left(8107<\mathrm{HJD}^{\prime}<8121\right)$, for which the effects of field rotation is much weaker. As shown in Figures B1 and B2, we find that the parallax measurements using first-half Spitzer data well consistent with the parallax using full Spitzer data. For the parallax measurements combining Spitzer observations, ground-based observations, and a VLTI constraint, we measured $\pi_{\mathrm{E}, \mathrm{N}}=$ $-0.479 \pm 0.057, \pi_{\mathrm{E}, \mathrm{E}}=0.206 \pm 0.024$ using first-half Spitzer data and $\pi_{\mathrm{E}, \mathrm{N}}=-0.430 \pm 0.055, \pi_{\mathrm{E}, \mathrm{E}}=0.186 \pm 0.024$ 
The Astrophysical Journal, 897:180 (12pp), 2020 July 10
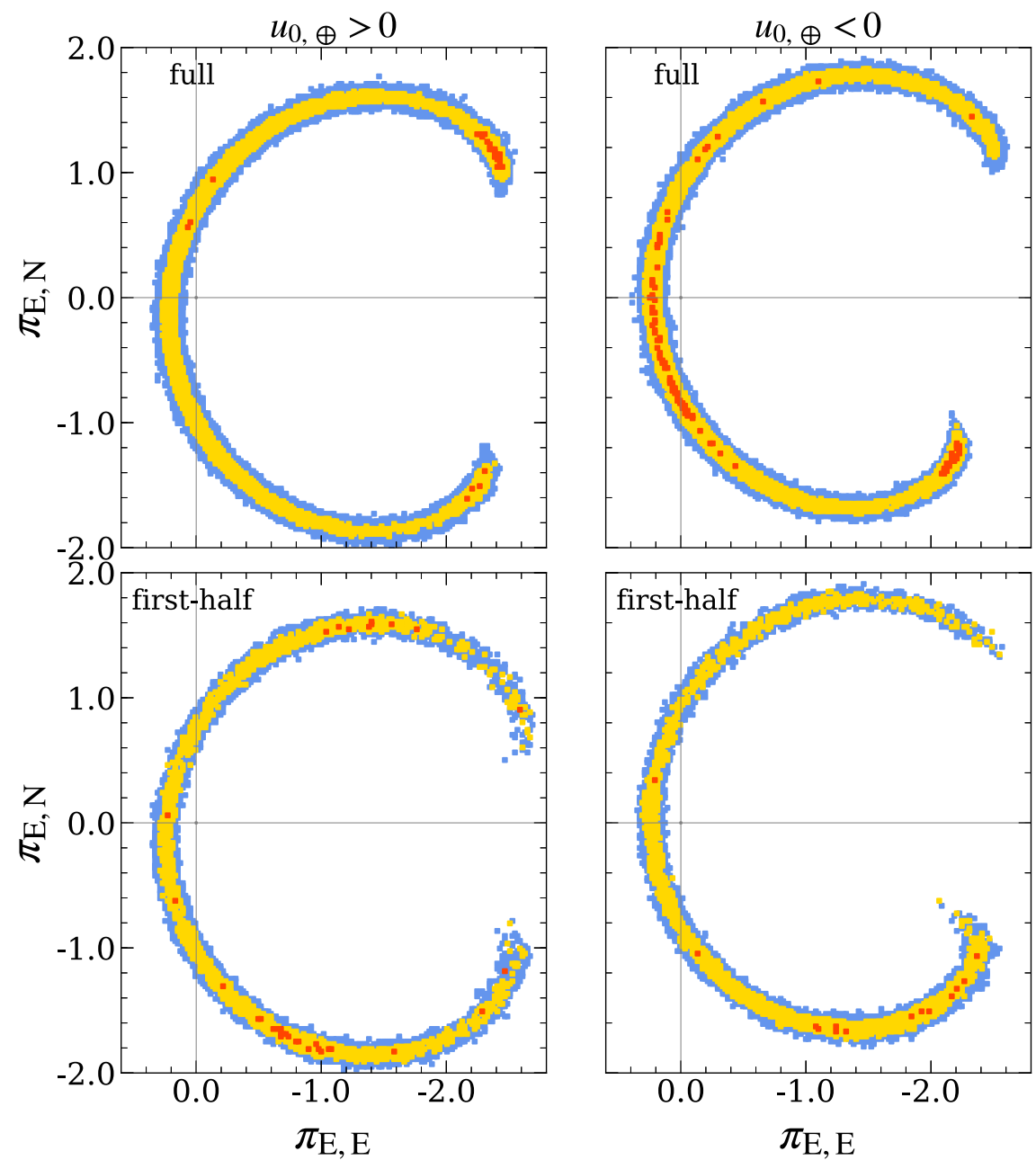

Figure B1. $\boldsymbol{\pi}_{\mathrm{E}}$ Likelihood distributions for "Spitzer-only" parallax using full and first-half $\left(8107<\mathrm{HJD}^{\prime}<8121\right)$ Spitzer data. The symbols are the same as those in Figure 2.
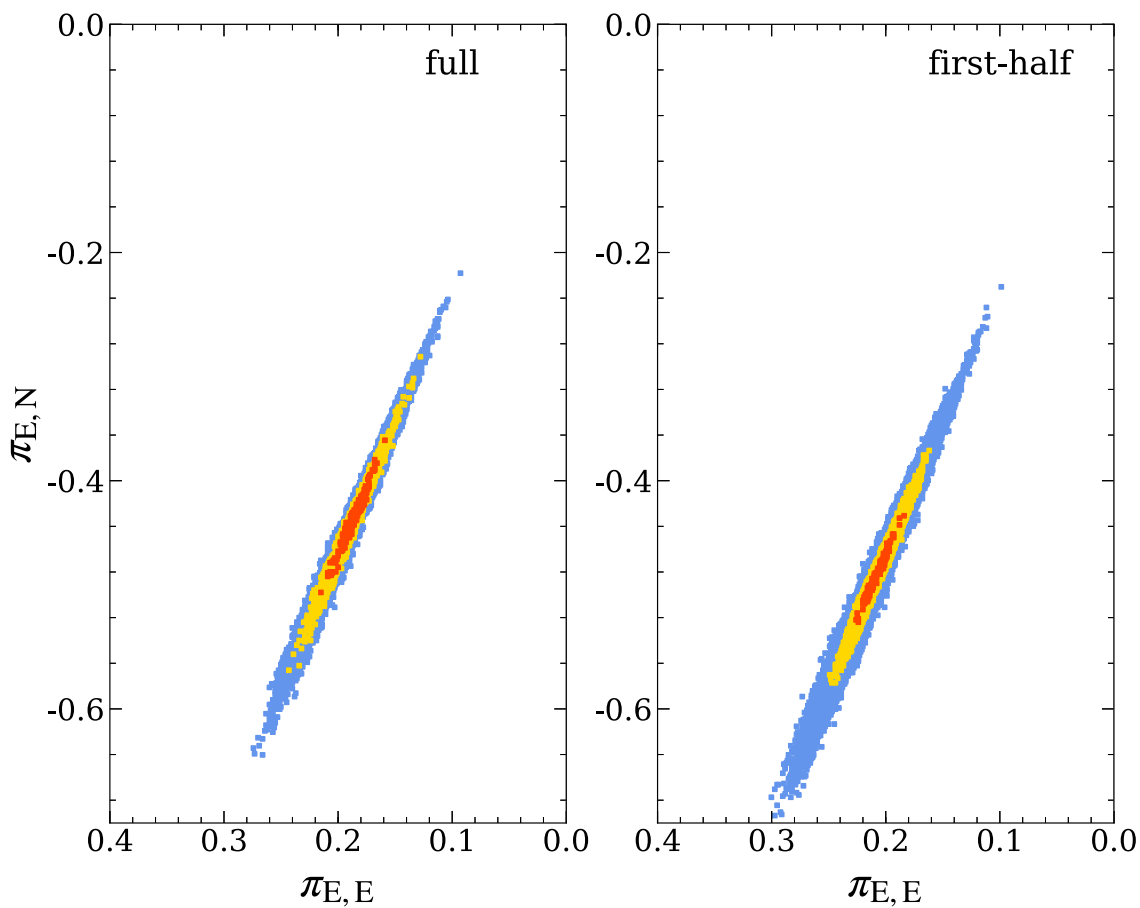

Figure B2. $\pi_{\mathrm{E}}$ Likelihood distributions for parallax combining Spitzer observations, ground-based observations, and a VLTI constraint using full (the left panel) and first-half Spitzer (the right panel) data. The symbols are similar to those in Figure 2. 
Table C1

Binary-lens Fitting Results with the VLTI Constraints

\begin{tabular}{|c|c|c|c|c|c|c|c|c|}
\hline \multirow{3}{*}{ Parameters } & \multicolumn{4}{|c|}{$s=0.935$} & \multicolumn{4}{|c|}{$s=0.975$} \\
\hline & \multicolumn{2}{|c|}{$u_{0, \oplus}>0$} & \multicolumn{2}{|c|}{$u_{0, \oplus}<0$} & \multicolumn{2}{|c|}{$u_{0, \oplus}>0$} & \multicolumn{2}{|c|}{$u_{0, \oplus}<0$} \\
\hline & w/o Lens Light & w/ Lens Light & w/o Lens Light & w/ Lens Light & w/o Lens Light & w/ Lens Light & w/o Lens Light & w/ Lens Light \\
\hline$t_{0, \oplus}\left(\mathrm{HJD}^{\prime}\right)$ & $8058.76(1)$ & $8058.76(1)$ & $8058.76(1)$ & $8058.76(1)$ & $8058.76(1)$ & $8058.76(1)$ & $8058.78(1)$ & $8058.76(1)$ \\
\hline$u_{0, \oplus}$ & $0.086(1)$ & $0.086(1)$ & $-0.094(1)$ & $-0.086(3)$ & $0.086(1)$ & $0.086(1)$ & -0.079 & -0.084 \\
\hline$t_{\mathrm{E}}$ & 27.82(16) & $27.82(16)$ & $28.10(41)$ & $28.04(67)$ & 27.81(17) & $27.84(16)$ & $29.61(48)$ & $28.15(37)$ \\
\hline$q\left(10^{-4}\right)$ & 1.1 & 1.1 & 1.1 & 1.1 & 1.1 & 1.1 & 1.1 & 1.1 \\
\hline$\alpha(\mathrm{rad})$ & 1.625 & 1.625 & 4.658 & 4.658 & 1.516 & 1.516 & 4.767 & 4.767 \\
\hline$\rho\left(10^{-3}\right)$ & $5.5(6)$ & $5.5(6)$ & $81(16)$ & $5.6(8)$ & $5.8(6)$ & $6.1(6)$ & $5.1(5)$ & $6.1(6)$ \\
\hline$\pi_{\mathrm{E}, \mathrm{N}}$ & $-0.427(57)$ & $-0.433(56)$ & $-0.014(337)$ & $-0.023(435)$ & $-0.445(56)$ & $-0.432(58)$ & $-0.004(292)$ & $-0.004(287)$ \\
\hline$\pi_{\mathrm{E}, \mathrm{E}}$ & $0.189(25)$ & $0.188(23)$ & $-0.003(76)$ & $-0.006(102)$ & $0.197(24)$ & $0.186(25)$ & $-0.001(66)$ & $-0.001(65)$ \\
\hline$\pi_{\mathrm{E}}$ & $0.467(62)$ & $0.472(61)$ & $0.015(346)$ & $0.024(447)$ & $0.486(61)$ & $0.470(63)$ & $0.004(299)$ & $0.004(295)$ \\
\hline$V_{\text {Стіо, } \mathrm{S}}$ & $14.15(1)$ & $14.15(1)$ & $14.17(2)$ & $14.16(3)$ & $14.15(1)$ & $14.15(1)$ & $14.17(3)$ & $14.17(3)$ \\
\hline$V_{\text {СтIо,в }}$ & $17.71(20)$ & $17.68(19)$ & $17.38(41)$ & $17.39(62)$ & $17.70(20)$ & $17.71(20)$ & $17.29(52)$ & $17.28(53)$ \\
\hline$H_{\mathrm{CTIO}, \mathrm{S}}$ & $12.02(1)$ & $12.02(1)$ & $12.03(2)$ & $12.03(3)$ & $12.02(1)$ & $12.02(1)$ & $12.03(3)$ & $12.03(3)$ \\
\hline$H_{\text {СтIO, В }}$ & $13.81(4)$ & $13.81(4)$ & $13.74(10)$ & $13.74(14)$ & $13.81(4)$ & $13.81(4)$ & $13.72(13)$ & 13.71(13) \\
\hline$\chi_{\text {Color }}^{2}$ & 0.026 & 0.019 & 41.722 & 41.891 & 0.027 & 0.058 & 41.643 & 41.274 \\
\hline$\chi_{\mathrm{VLTI}}^{2}$ & 0.040 & 0.114 & 0.074 & 0.028 & 0.024 & 0.026 & 0.202 & 0.390 \\
\hline$\chi_{\text {total }}^{2} /$ dof & $575.1 / 554$ & $575.2 / 554$ & $618.9 / 574$ & $619.0 / 574$ & $575.3 / 574$ & $575.2 / 574$ & $617.8 / 574$ & $617.2 / 574$ \\
\hline
\end{tabular}

using full Spitzer data, which are in agreement with each other within $1 \sigma$. This demonstrates that the effects of field rotation on Spitzer photometry are insignificant.

\section{Appendix C The Planetary Model}

Nucita et al. (2018) reported a short-duration anomaly near the peak of the event, indicating that the lens star has a planetary companion with planet-host mass ratio $q=1.1 \times 10^{-4}$. To double check the parallax measurements, we fit the event with the binary-lens model. Besides the three parameters $t_{0}, u_{0}, t_{\mathrm{E}}$ introduced in Section 3.1, the binary-lens model has four other parameters $(s, q, \alpha, \rho)$. Here, $q$ is the companion-host mass ratio, $s$ is the companion-host projected separation in units of $\theta_{\mathrm{E}}$, $\alpha$ is the angle between the source trajectory and the binary axis in the lens plane, and $\rho$ is the source radius normalized to $\theta_{\mathrm{E}}$.

We fix $(s, q, \alpha)$ as the best-fit values shown in Nucita et al. (2018). We include the satellite parallax and the constraints from VLTI-GRAVITY. We consider the so-called "minorimage perturbation degeneracy" (e.g., Koshimoto et al. 2017; Han et al. 2018; Calchi Novati et al. 2019) found by Nucita et al. (2018): $s=0.935 \pm 0.004$ and $s=0.975 \pm 0.004$. Table $\mathrm{C} 1$ shows the best-fit parameters. For all the solutions, the resulting parallax is consistent with the results of the singlelens model within $1 \sigma$. Thus, different models (single-lens and binary-lens) do not have significant influence on the parallax and thus the mass measurements.

\section{ORCID iDs}

Weicheng Zang (iD https://orcid.org/0000-0001-6000-3463 Subo Dong (ib https://orcid.org/0000-0002-1027-0990 Sebastiano Calchi Novati (ib https://orcid.org/0000-00027669-1069

Ping Chen (iD https://orcid.org/0000-0003-0853-6427 Hongjing Yang (iD https://orcid.org/0000-0003-0626-8465 Shun-Sheng Li (i) https://orcid.org/0000-0001-9952-7408 Shude Mao (i) https://orcid.org/0000-0001-8317-2788 Sean Carey (iD https://orcid.org/0000-0002-0221-6871
Dax L. Feliz (iD https://orcid.org/0000-0002-2457-7889 T. Jayasinghe (1) https://orcid.org/0000-0002-6244-477X Robert Mutel (1) https://orcid.org/0000-0003-1511-6279 Yossi Shvartzvald (iD https://orcid.org/0000-0003-1525-5041 Wei Zhu (iD https://orcid.org/0000-0003-4027-4711

\section{References}

Alcock, C., Allsman, R. A., Alves, D. R., et al. 2001, Natur, 414, 617 Bailer-Jones, C. A. L., Rybizki, J., Fouesneau, M., Mantelet, G., \& Andrae, R. 2018, AJ, 156, 58

Batista, V., Beaulieu, J.-P., Bennett, D. P., et al. 2015, ApJ, 808, 170

Beaulieu, J.-P., Batista, V., Bennett, D. P., et al. 2018, AJ, 155, 78

Bennett, D. P., Anderson, J., \& Gaudi, B. S. 2007, ApJ, 660, 781

Bennett, D. P., Becker, A. C., Calitz, J. J., et al. 2002, arXiv:astro-ph/0207006

Bennett, D. P., Bhattacharya, A., Anderson, J., et al. 2015, ApJ, 808, 169

Bennett, D. P., Bhattacharya, A., Beaulieu, J.-P., et al. 2020, AJ, 159, 68

Bhattacharya, A., Beaulieu, J. P., Bennett, D. P., et al. 2018, AJ, 156, 289

Calchi Novati, S., Gould, A., Udalski, A., et al. 2015a, ApJ, 804, 20

Calchi Novati, S., Gould, A., Yee, J. C., et al. 2015b, ApJ, 814, 92

Calchi Novati, S., Skowron, J., Jung, Y. K., et al. 2018, AJ, 155, 261

Calchi Novati, S., Suzuki, D., Udalski, A., et al. 2019, AJ, 157, 121

Cardelli, J. A., Clayton, G. C., \& Mathis, J. S. 1989, ApJ, 345, 245

DePoy, D. L., Atwood, B., Belville, S. R., et al. 2003, Proc. SPIE, 4841, 827

Dong, S., Carey, S., Gould, A., \& Zhu, W. 2017, First Mass Measurement of a 'Domestic' Microlens, Spitzer Proposal, ID \#13250

Dong, S., Gould, A., Udalski, A., et al. 2009, ApJ, 695, 970

Dong, S., Mérand, A., Delplancke-Ströbele, F., et al. 2019, ApJ, 871, 70

Dong, S., Udalski, A., Gould, A., et al. 2007, ApJ, 664, 862

Fukui, A., Suzuki, D., Koshimoto, N., et al. 2019, AJ, 158, 206

Gaia Collaboration, Brown, A. G. A., Vallenari, A., et al. 2018, A\&A, 616, A1

Gaudi, B. S. 2012, ARA\&A, 50, 411

Gould, A. 1992, ApJ, 392, 442

Gould, A. 1994, ApJL, 421, L75

Gould, A. 1995, ApJL, 441, L21

Gould, A. 2000a, ApJ, 535, 928

Gould, A. 2000b, ApJ, 542, 785

Gould, A. 2004, ApJ, 606, 319

Gould, A. 2019, JKAS, 52, 121

Gould, A., Carey, S., \& Yee, J. 2013, Spitzer Microlens Planets and Parallaxes, Spitzer Proposal, ID 10036

Gould, A., Carey, S., \& Yee, J. 2014, Galactic Distribution of Planets from Spitzer Microlens Parallaxes, Spitzer Proposal, ID \#11006

Gould, A., Carey, S., \& Yee, J. 2016, Galactic Distribution of Planets Spitzer Microlens Parallaxes, Spitzer Proposal, ID \#13005

Gould, A., \& Loeb, A. 1992, ApJ, 396, 104 
Gould, A., Ryu, Y.-H., Calchi Novati, S., et al. 2020, JKAS, 53, 9

Gould, A., Udalski, A., Monard, B., et al. 2009, ApJL, 698, L147

Gould, A., Yee, J., \& Carey, S. 2015a, Galactic Distribution of Planets From High-Magnification Microlensing Events, Spitzer Proposal, ID \#12013

Gould, A., Yee, J., \& Carey, S. 2015b, Degeneracy Breaking for K2 Microlens Parallaxes, Spitzer Proposal, ID \#12015

Gould, A., Yee, J., Carey, S., \& Shvartzvald, Y. 2018, The Galactic Distribution of Planets via Spitzer Microlensing Parallax, Spitzer Proposal, ID \#14012

Gravity Collaboration, Abuter, R., Accardo, M., et al. 2017, A\&A, 602, A94

Han, C., Bond, I. A., Gould, A., et al. 2018, AJ, 156, 226

Han, C., Yee, J. C., Udalski, A., et al. 2019, AJ, 158, 102

Hardy, S. J., \& Walker, M. A. 1995, MNRAS, 276, L79

Hirao, Y., Bennett, D. P., Ryu, Y.-H., et al. 2020, arXiv:2004.09067

Holz, D. E., \& Wald, R. M. 1996, ApJ, 471, 64

Jiang, G., DePoy, D. L., Gal-Yam, A., et al. 2004, ApJ, 617, 1307

Jung, Y. K., Gould, A., Udalski, A., et al. 2019, AJ, 158, 28

Jung, Y. K., Han, C., Udalski, A., et al. 2018, ApJ, 863, 22

Kennedy, G. M., \& Kenyon, S. J. 2008, ApJ, 673, 502

Koshimoto, N., \& Bennett, D. 2019, arXiv:1905.05794

Koshimoto, N., Udalski, A., Beaulieu, J. P., et al. 2017, AJ, 153, 1

Kozłowski, S., Woźniak, P. R., Mao, S., \& Wood, A. 2007, ApJ, 671, 420

Mao, S. 2012, RAA, 12, 947

Mao, S., \& Paczynski, B. 1991, ApJL, 374, L37

Mao, S., Smith, M. C., Woźniak, P., et al. 2002, MNRAS, 329, 349

Mróz, P., Ryu, Y.-H., Skowron, J., et al. 2018, AJ, 155, 121
Mróz, P., Udalski, A., Bennett, D. P., et al. 2019, A\&A, 622, A201

Mróz, P., Udalski, A., Skowron, J., et al. 2017, Natur, 548, 183

Nemiroff, R. J., \& Wickramasinghe, W. A. D. T. 1994, ApJL, 424, L21

Nucita, A. A., Licchelli, D., de Paolis, F., et al. 2018, MNRAS, 476, 2962

Paczyński, B. 1986, ApJ, 304, 1

Poindexter, S., Afonso, C., Bennett, D. P., et al. 2005, ApJ, 633, 914

Refsdal, S. 1966, MNRAS, 134, 315

Ryu, Y.-H., Yee, J. C., Udalski, A., et al. 2018, AJ, 155, 40

Shappee, B. J., Prieto, J. L., Grupe, D., et al. 2014, ApJ, 788, 48

Shvartzvald, Y., Yee, J. C., Calchi Novati, S., et al. 2017, ApJL, 840, L3

Shvartzvald, Y., Yee, J. C., Skowron, J., et al. 2019, AJ, 157, 106

Street, R. A., Udalski, A., Calchi Novati, S., et al. 2016, ApJ, 819, 93

Sumi, T., Kamiya, K., Bennett, D. P., et al. 2011, Natur, 473, 349

Udalski, A., Ryu, Y.-H., Sajadian, S., et al. 2018, AcA, 68, 1

Udalski, A., Yee, J. C., Gould, A., et al. 2015, ApJ, 799, 237

Vandorou, A., Bennett, D. P., Beaulieu, J.-P., et al. 2019, arXiv:1909.04444

Witt, H. J., \& Mao, S. 1994, ApJ, 430, 505

Wyrzykowski, Ł., Kostrzewa-Rutkowska, Z., Skowron, J., et al. 2016 , MNRAS, 458, 3012

Yee, J. C. 2015, ApJL, 814, L11

Yee, J. C., Udalski, A., Sumi, T., et al. 2009, ApJ, 703, 2082

Yoo, J., DePoy, D. L., Gal-Yam, A., et al. 2004, ApJ, 603, 139

Zang, W., Shvartzvald, Y., Wang, T., et al. 2020, ApJ, 891, 3

Zhu, W., Calchi Novati, S., Gould, A., et al. 2016, ApJ, 825, 60

Zhu, W., Udalski, A., Calchi Novati, S., et al. 2017a, AJ, 154, 210

Zhu, W., Udalski, A., Huang, C. X., et al. 2017b, ApJL, 849, L31 